\title{
ON RELIABILITY PREDICTION AND SEMI-RENEWAL PROCESSES
}

\author{
MICHAEL TORTORELLA, * Rutgers University
}

\begin{abstract}
We analyze the notion of 'reliability prediction' by studying in detail a key property that is tacitly assumed to make reliability prediction possible. The analysis leads in turn to a special type of point process for which the connection of future to past can be explicitly displayed. In this type of process, the semi-renewal process, all finitedimensional distributions are completely determined by the distribution of the time to the first event in the process. The theory provides a heretofore unappreciated unification of the two most commonly used reliability prediction models for maintained systems, namely, the renewal and revival processes. We show that familiar results from renewal theory extend and generalize to semi-renewal processes.
\end{abstract}

Keywords: Renewal; relevation; bad-as-old; record value process; semigroup

2010 Mathematics Subject Classification: Primary 60G55; 90B25

\section{Introduction}

\subsection{Rationale}

Humans are notoriously bad about predicting the future. On what basis, then, does the activity of reliability prediction in engineering deserve any serious consideration? Close analysis of how reliability prediction is usually performed reveals that the common feature in all such exercises is that they use assumptions (sometimes, but usually not, explicitly stated) about how the future resembles the past. In this paper we explore an explicit formulation of such an assumption that leads to an interesting new class of stochastic point processes.

\subsection{Scope}

The two most commonly used stochastic point process models for the reliability of a maintained system are the renewal process [7, p. 167] and the process that has been called the 'minimal repair' or 'bad-as-old' process [1]. A key property shared by these two types of processes is the following.

Property 1. If you know the distribution of the time to the first event in the process then you know all the finite-dimensional distributions of the process.

That is, the distribution of the time to the first event in the process completely determines the probabilistic structure of the entire process.

For the renewal process, this is obvious. For the nonhomogeneous Poisson process (with which the minimal repair process is often conflated), see [12, Equation 2.42], to see that the expression for the joint distribution of any collection of intervals in the process contains

Received 2 October 2008; revision received 4 February 2010.

* Current address: RUTCOR 148, Rutgers University, 640 Bartholomew Road, Piscataway, NJ 08854, USA.

Email address: mtortore@ rci.rutgers.edu 
only quantities that can be obtained from the distribution of the time to the first event in the process. (Here, by 'interval' we mean the length of time between two successive events in the process.) This property is a strong assertion about how the future is connected to the past. It says, essentially, that if we have only one piece of information about the past then we know everything about the future evolution of the process (in the probabilistic sense). (For the purposes of this discussion, let us agree to fix 'the present' as the time of occurrence of the first event in the process.)

It is possible to imagine several ways of formalizing Property 1 . In this paper we explore one such way. We study stochastic point processes for which the conditional distribution of any interval, given the previous intervals, is obtained as the image of the distribution of the first interval in the process under a map (operator) chosen, via the values of conditioning variables, from a given set, which is later specialized to be a semigroup, of operators. In practical terms, this procedure says that there is a rule (chosen from some structured set of rules) that enables us to obtain (the distribution of) any interval if we know only the occurrence times of the previous events and the distribution of the first interval. The dispersion in any interval is controlled by the dispersion in the first interval. A pure (without distortion) time-shift model, useful for gaining understanding, is the renewal process, a familiar special case of the general theory presented below.

\subsection{Background}

For applications, semi-renewal processes are also useful in modeling repair processes that may be imperfect or that may be intermediate between renewal (or complete overhaul) and minimal repair. Brown and Proschan [5] and Kijima [8] have previously studied related models, the former for which a repair is either a renewal or minimal according to an independent toss of a coin at each failure time, and the latter for repairs that return the hazard rate of the failure time of the repaired unit to some multiple (between 0 and 1) of its value at the time of the unit's previous failure. Baxter and Chlouverakis [2], [3] studied models of clumsy repair actions that may introduce additional faults during a repair. In this case, the hazard rate of the time to the next failure of the repaired item may be greater than that for a new unit and greater than its value at the time of the unit's previous failure. Baxter et al. [4] studied a general model for equipment condition after repair that includes as special cases all the models above.

\subsection{Synopsis}

From the point of view of theory, the point processes studied here generalize both the renewal process and the revival process (itself a generalization of the nonhomogeneous Poisson process; see [13]). Besides highlighting additional common features of these processes, the generalization shows that many results and techniques resembling those of renewal theory can be more widely applied to yield new results and new ways of looking at known results.

\section{The semi-renewal process}

\subsection{Introduction}

The generalization studied here will be called a 'semi-renewal process'. The terminology is intended to convey a notion that everything that happens in the future is (probabilistically) constrained to develop within the confines of a framework defined by a given set of (deterministic) maps and the distribution of the time to the first event. In any point process, any interval is 'linked' to the previous intervals by an expression for its conditional distribution, given the lengths of the previous intervals, which is obtained from the finite-dimensional distributions of 
the interval (or time) representation of the process. We will introduce the framed point process in which this linkage is expressed in a particular way that is amenable to further analysis. In more mathematical terms, for every point process (whose intervals are denoted by $X_{1}, X_{2}, \ldots$ ), the conditional distribution $\mathrm{P}\left\{X_{n+1} \leq x \mid X_{1}=x_{1}, \ldots, X_{n}=x_{n}\right\}$ can be understood as a map from $\mathbb{R}_{+}^{n}$ into the set $L$ of life distributions that 'links $X_{n+1}$ with the previous $X \mathrm{~s}$ '. In a framed point process, this map factors through the space $\left(L^{L}\right)^{\mathbb{R}^{n}}$ of frames, producing a special class of point processes that generalizes the renewal process and the revival process and for which some members of the class can be further analyzed.

The connection with 'prediction' is that the frame embodies the assumptions we make about how the future is linked to the past. This study shows one way to make these assumptions explicit.

\subsection{Framed point processes}

This section provides definitions and examples of framed point processes. Throughout, we will let $L$ denote the set of univariate life distributions (a life distribution is a cumulative distribution function whose left-hand limit at 0 is 0 ). We denote by $L^{L}$ the set of all maps from $L$ into itself. Let $\mathbb{R}^{n}$ represent the $n$-dimensional Euclidean space, and let $\mathbb{R}_{+}^{n}$ be its positive orthant $\left\{\left(x_{1}, \ldots, x_{n}\right): x_{i} \geq 0, i=1, \ldots, n\right\}$.

Definition. For each $n=1,2, \ldots$, let $T_{n}: \mathbb{R}_{+}^{n} \rightarrow L^{L}$. The set $\mathbb{T}=\left\{T_{n}: n=1,2, \ldots\right\}$ is called a frame.

In a frame, each $T_{n}$ maps $n$-vectors into transformations of $L$ into itself. That is, for each positive integer $n$ and each $\left(x_{1}, \ldots, x_{n}\right) \in \mathbb{R}_{+}^{n}, T_{n}\left(x_{1}, \ldots, x_{n}\right)$ is a map that takes a life distribution into (possibly another) life distribution.

Definition. A point process with intervals $\left\{X_{1}, X_{2}, \ldots\right\}$ is said to be framed if there is a frame $\mathbb{T}$ for which

$$
\mathrm{P}\left\{X_{n+1} \leq x \mid X_{1}=x_{1}, \ldots, X_{n}=x_{n}\right\}=\left[T_{n}\left(x_{1}, \ldots, x_{n}\right) F\right](x),
$$

where $F$ is the distribution of $X_{1}$.

The prescription for determining the conditional distribution of an interval in a framed point process is as follows. Take the values of the previous intervals and select the proper size frame element $(n)$. Insert the interval values into the frame element and operate with the resulting map on the distribution of the time to the first event. The end product is another life distribution that is the desired conditional distribution.

\subsection{Semi-renewal process definition}

While the framed point process does possess Property 1, which states that (the distribution of) any interval is determined by the frame and the distribution of the first interval, the generality of the frame as defined above makes further analysis difficult. The following special case tempers this difficulty while preserving the important notion that the future stochastic description of the process is mediated by a known (special type of) frame, with the randomness in this description coming essentially from the dispersion in the first interval.

Definition. A framed point process is said to be a semi-renewal process if its frame depends only on the sum of its arguments, i.e. $T_{n}\left(x_{1}, \ldots, x_{n}\right)=T\left(x_{1}+\cdots+x_{n}\right)$, where $T$ is a semigroup (with identity) of operators in $L^{L}$ (that is, for each $x, T(x)$ is a map taking $L$ into itself, $T(0)$ is the identity map, and $T\left(x_{1}+x_{2}\right)=T\left(x_{1}\right) T\left(x_{2}\right)$ for all nonnegative $x_{1}$ and $\left.x_{2}\right)$. 
The semi-renewal process is a special case of the counting process governed by nonnegative Markovian increments introduced by Kijima and Sumita [9]. In the semi-renewal process, however, some explicit computations, including those in (8) and Theorems 3 and 5, below, are possible that are not available in the more general case. Note also that the definition involves only the one-dimensional distributions of the process; no assumptions about covariances or any other higher-order properties are imposed.

Letting $S_{n}=X_{1}+\cdots+X_{n}$, for a semi-renewal process, we obtain, for each $n=0,1,2, \ldots$,

$$
\begin{aligned}
\mathrm{P}\left\{X_{n+1} \leq x \mid X_{1}=x_{1}, \ldots, X_{n}=x_{n}\right\} & =\left[T\left(x_{1}+\cdots+x_{n}\right) F\right](x) \\
& =\mathrm{P}\left\{X_{n+1} \leq x \mid S_{n}=x_{1}+\cdots+x_{n}\right\},
\end{aligned}
$$

or, in short,

$$
\mathrm{P}\left\{X_{n+1} \leq x \mid S_{n}=u\right\}=T_{u} F(x),
$$

where, for convenience, we have written $u=x_{1}+\cdots+x_{n}$ and $T_{u}$ instead of $T(u)$. The invariance of the right-hand side of (2) with $n$ indicates that we have captured an important aspect of reliability prediction models that we have been focusing on: it does not matter how many events (failures) have taken place, or when they took place (except for the most recent one), the mechanism that takes us to the next inter-failure time is always the same (except for possible dependence on the current failure occurrence time). Stating it another way, and recalling that we have defined 'the present' as time $X_{1}=S_{1}$ of the occurrence of the first failure, we see that once we have made the assumption (embodied in (1) when $n=1$ ) about how the future is linked to the past, then the further evolution of the process is brought about by the very same mechanism, or assumptions. The mechanism that gets us from $S_{n}$ to $X_{n+1}$ is always the same as the mechanism that gets us from $S_{1}$ to $X_{2}$, and now we can make probability statements about the future reliability of the maintained system modeled by this process because the stochastic part of the future is governed by the stochastic part of $X_{1}$, which we assume we know (or can estimate).

\subsection{Examples}

2.4.1. Renewal process, almost. Via (1) and (2), we see that the semi-renewal process generated by the trivial semigroup $T_{u}=I$ for every $u$ ( $I$ is the identity operator) satisfies all the conditions required of a renewal process except the mutual independence of the sequence $\left\{X_{1}, X_{2}, \ldots\right\}$. Inductive application of (2) shows that each $X_{n}$ has the same distribution and that $X_{n}$ is independent of $S_{n-1}$. However, the following example, due to Norman A. Marlow, shows that $\left\{X_{1}, X_{2}, \ldots\right\}$ need not be mutually stochastically independent.

Example. Let $0<p<1$, and choose $h$ such that $0<2 h p<1$ and $0<2(1-h) p<1$. Define three random variables $X, Y$, and $Z$ by the joint distribution shown in Table 1 . Then $X, Y$, and $Z$ have the following properties:

1. they are nonnegative and identically distributed, each assuming the values 1 or 0 with probabilities $p$ and $1-p$, respectively;

2. $X$ and $Y$ are independent;

3. $Z$ is independent of $X+Y$;

4. $X+Y+Z$ has the same distribution as it would if $X, Y$, and $Z$ were mutually independent; but

5. $X, Y$, and $Z$ are not mutually independent. 
TABLe 1.

\begin{tabular}{cccc}
\hline$X$ & $Y$ & $Z$ & Probability \\
\hline 1 & 1 & 1 & $p^{3}$ \\
1 & 1 & 0 & $p^{2}(1-p)$ \\
1 & 0 & 1 & $2(1-h) p^{2}(1-p)$ \\
1 & 0 & 0 & $p(1-p)[1-2(1-h) p]$ \\
0 & 1 & 1 & $2 h p^{2}(1-p)$ \\
0 & 1 & 0 & $p(1-p)(1-2 h p)$ \\
0 & 0 & 1 & $p(1-p)^{2}$ \\
0 & 0 & 0 & $(1-p)^{3}$ \\
\hline
\end{tabular}

The material in the remainder of this paper shows that many of the familiar results of renewal theory continue to hold if only the $n$th interevent time interval is independent of the occurrence time of the previous event, and not necessarily of all the previous interevent time intervals.

2.4.2. Revival process. If, for $u, x \geq 0$,

$$
T_{u} F(x)=\frac{F(x+u)-F(u)}{1-F(u)},
$$

then the corresponding semi-renewal process is a revival process. It is straightforward to verify the semigroup property for (3), and then (2) is the weak minimal repair property that defines the revival process [13]. This process does not necessarily have independent increments, so it is not necessarily a Poisson process even though all its finite-dimensional distributions are Poisson.

2.4.3. Record value process. If

$$
T_{u} F(x)=F(x+u)
$$

then the corresponding semi-renewal process $\left\{S_{n}: n=1,2, \ldots\right\}$ is the record value process of a set of independent and identically distributed (i.i.d.) nonnegative random variables, or

$$
S_{n}=\max \left\{Y_{1}, \ldots, Y_{n}\right\}
$$

where the $Y_{1}, Y_{2}, \ldots$ are i.i.d. and $X_{n}=S_{n}-S_{n-1}$ with $S_{0}=0$.

\section{The semi-renewal argument}

The principle that makes the rest of the theory work is that the standard renewal argument (that, conditional on the value of the first interval, the remainder of the process is still a renewal process with the same inter-renewal time distribution) generalizes to the semi-renewal case.

Theorem 1. Let $\left\{X_{n}: n=1,2, \ldots\right\}$ be a semi-renewal process under the semigroup $T$, and let the distribution of $X_{1}$ be $F$. Then, conditional on $X_{1}=u$, the remainder $\left\{X_{n}: n=2,3, \ldots\right\}$ is a semi-renewal process under the same semigroup $T$ and with the distribution of its first interval (i.e. $X_{2}$ ) being $T_{u} F$. 
Proof. Whenever $X_{1}=u$, let $X_{1}^{\prime}=X_{2}, X_{2}^{\prime}=X_{3}$, etc. Let $\mathrm{P}_{u}$ denote the conditional probability measure $\mathrm{P}_{u}(A)=\mathrm{P}\left\{A \mid X_{1}=u\right\}$. Then

$$
\begin{aligned}
& \mathrm{P}_{u}\left\{X_{n+1}^{\prime} \leq x \mid X_{1}^{\prime}=u_{1}, \ldots, X_{n}^{\prime}=u_{n}\right\} \\
& \quad=\mathrm{P}\left\{X_{n+2} \leq x \mid X_{2}=u_{1}, \ldots, X_{n+1}=u_{n}, X_{1}=u\right\} \\
& \quad=T_{u_{1}+\cdots+u_{n}+u} F(x) \\
& \quad=T_{u} T_{u_{1}+\cdots+u_{n}} F(x) \\
& \quad=T_{u_{1}+\cdots+u_{n}} T_{u} F(x),
\end{aligned}
$$

which establishes the result.

\subsection{Counting the number of events in a semi-renewal process}

As an application of the semi-renewal argument, we will show how to obtain the expected number of events in a semi-renewal process in a given time interval. Let $V$ denote the unit step function at 0 , and set $N(F, t)=\sum_{n=1}^{\infty} V\left(t-S_{n}\right)$ and $M(F, t)=\mathrm{E} N(F, t)$. Using Theorem 1 , we obtain

$$
\mathrm{E}\left[N(F, t) \mid X_{1}=u\right]= \begin{cases}0, & t<u, \\ 1+\mathrm{E} N\left(T_{u} F, t-u\right), & t \geq u,\end{cases}
$$

so that, using the law of total probability,

$$
M(F, t)=F(t)+\int_{0}^{t} M\left(T_{u} F, t-u\right) \mathrm{d} F(u) .
$$

When $T_{u}=I$ for every $u$, then (5) is the familiar equation for the expected number of events in an ordinary renewal process in the time interval $[0, t]$. If $T_{u}$ is the semigroup from (3) then it is easy to verify that $M(F, t)=-\log [1-F(t)]$ is a solution of (5). If $T_{u}$ is the semigroup from (4) then we have

$$
\begin{aligned}
M(F, t) & =\sum_{n=1}^{\infty} \mathrm{P}\left\{S_{n} \leq t\right\} \\
& =F(t)+\sum_{n=2}^{\infty} \int_{0}^{\infty} \mathrm{P}\left\{S_{n} \leq t \mid S_{n-1}=u\right\} \mathrm{dP}\left\{S_{n-1} \leq u\right\} \\
& =F(t)+\sum_{n=2}^{\infty} \int_{0}^{t} \mathrm{P}\left\{X_{n} \leq t-u \mid S_{n-1}=u\right\} \mathrm{dP}\left\{S_{n-1} \leq u\right\} \\
& =F(t)+\sum_{n=2}^{\infty} \int_{0}^{t} T_{u} F(t-u) \mathrm{dP}\left\{S_{n-1} \leq u\right\} \\
& =F(t)+\sum_{n=2}^{\infty} \int_{0}^{t} F(t) \mathrm{dP}\left\{S_{n-1} \leq u\right\} \\
& =F(t)+F(t) \sum_{n=1}^{\infty} \mathrm{P}\left\{S_{n} \leq t\right\} \\
& =F(t)[1+M(F, t)],
\end{aligned}
$$


so that, for the record values process, we obtain

$$
M(F, t)=\frac{F(t)}{1-F(t)} .
$$

Of course, we could always substitute (6) into (5) to verify that (6) is a solution of (5), but the derivation shows another way to obtain the familiar result (6).

In fact, as in renewal theory, we will see that further computational developments for more general integral equations of semi-renewal type (see the next section) hinge on whether we can obtain a solution for the basic semi-renewal equation (5). In cases represented by (3) and (4) we are able to obtain these in closed form, but even in the ordinary renewal process case, closed-form solutions to (5) are rare.

Before moving on, we will establish some continuity and differentiability properties of (5).

Theorem 2. Suppose that $u \rightsquigarrow T_{u}$ is a continuous semigroup [14] having $\left|T_{u} F(t-u)\right| \leq F(t)$ for all $0 \leq u \leq t$. If $F(\cdot)$ is continuous then $M(F, \cdot)$ is continuous. If $F(\cdot)$ is continuously differentiable then $M(F, \cdot)$ is continuously differentiable.

Proof. Define

$$
F^{[2]}(t)=\int_{0}^{t} T_{u} F(t-u) \mathrm{d} F(u) \quad \text { and } \quad F^{[n+1]}(t)=\int_{0}^{t} T_{u} F^{[n]}(t-u) \mathrm{d} F(u)
$$

for $n=2,3, \ldots$ In general, this leads to

$$
F^{[n+1]}(t)=\int_{0}^{t} \cdots \int_{0}^{t} T_{u_{1}+\cdots+u_{n}} F\left(t-u_{1}-\cdots-u_{n}\right) \mathrm{d} F\left(u_{1}\right) \cdots \mathrm{d} F\left(u_{n}\right),
$$

from which it follows by induction that $\left|F^{[n]}(t)\right| \leq F(t)^{n}$ for $n=1,2, \ldots$ Then the series

$$
M(F, t)=\sum_{n=1}^{\infty} \mathrm{P}\left\{S_{n} \leq t\right\}=\sum_{n=1}^{\infty} F^{[n]}(t)
$$

converges geometrically, hence uniformly, and both assertions follow from this fact.

Note that all the semigroups of Section 2.4 satisfy these conditions.

\section{Integral equations of semi-renewal type}

\subsection{Explicit solutions}

As in standard renewal theory, many applications reduce to the derivation and solution of a more general integral equation for the quantities under study. We will show some examples in Section 4.3, below. The general form of these equations in the semi-renewal case includes the semigroup parameter as the variable of integration. This is as follows:

$$
r(F, t)=h(F, t)+\int_{0}^{t} r\left(T_{u} F, t-u\right) \mathrm{d} F(u),
$$

where $h$ is a given function that may depend on $F$ (in applications, it usually does), and $r$ is unknown and to be solved for. 
Theorem 3. Equation (7) has a solution that is given by

$$
r(F, t)=h(F, t)+\int_{0}^{t} h\left(T_{u} F, t-u\right) \mathrm{d} M(F, u) .
$$

Proof. We use (5) and the semigroup property of $T$ to obtain

$$
\begin{aligned}
r(F, t) & -h(F, t) \\
= & \int_{0}^{t}\left[h\left(T_{u} F, t-u\right)+\int_{0}^{t-u} h\left(T_{v}\left(T_{u} F\right), t-u-v\right) \mathrm{d} M(F, v)\right] \mathrm{d} F(u) \\
= & \int_{0}^{t} h\left(T_{u} F, t-u\right) \mathrm{d} F(u)+\int_{0}^{t} \int_{0}^{t-u} h\left(T_{u+v} F, t-(u+v)\right) \mathrm{d} M(F, v) \mathrm{d} F(u) \\
= & \int_{0}^{t} h\left(T_{u} F, t-u\right) \mathrm{d} F(u)+\int_{0}^{t} h\left(T_{s} F, t-s\right) \mathrm{d}_{s}\left[\int_{0}^{s} M(F, s-x) \mathrm{d} F(x)\right] \\
= & \int_{0}^{t} h\left(T_{u} F, t-u\right) \mathrm{d} F(u)+\int_{0}^{t} h\left(T_{s} F, t-s\right) \mathrm{d}[M(F, s)-F(s)] \\
= & \int_{0}^{t} h\left(T_{u} F, t-u\right) \mathrm{d} M(F, u),
\end{aligned}
$$

which completes the proof.

Now we present some simple sufficient conditions for the solution (8) to be unique.

Definition. A function $h: L \times \mathbb{R} \rightarrow \mathbb{R}$ is called locally semi-bounded if these exists $B<\infty$ (maybe depending on $F$ and/or $t$ ) for which $\left|h\left(T_{u} F, t-u\right)\right| \leq B$ for all $u \in[0, t]$.

Note that, when $T_{u}=I$, local semi-boundedness reduces to local boundedness [7, p. 184], a condition sufficient for the uniqueness of the solution to the ordinary integral equation of renewal type.

Theorem 4. If $h$ is locally semi-bounded then the solution (8) of the integral equation of semirenewal type is unique in the class of locally semi-bounded functions.

Proof. It suffices to show that the only locally semi-bounded solution to the homogeneous form of (7) is identically 0 . Consider the homogeneous equation

$$
R(F, t)=\int_{0}^{t} R\left(T_{u} F, t-u\right) \mathrm{d} F(u)
$$

From (9), for every positive integer $n$, we have

$$
\begin{array}{rl}
R(F, t)=\int_{0}^{t} \int_{0}^{t-x_{1}} \cdots \int_{0}^{t-x_{1}-\cdots-x_{n}} & R\left(T_{u+x_{1}+\cdots+x_{n}} F, t-\left(u+x_{1}+\cdots+x_{n}\right)\right) \\
& \times \mathrm{d} F(u) \mathrm{d} F\left(x_{n}\right) \cdots \mathrm{d} F\left(x_{1}\right) .
\end{array}
$$

Because $R$ is restricted to be locally semi-bounded, we have, for some $B<\infty$ and every positive integer $n$,

$$
|R(F, t)| \leq B \int_{0}^{t} \int_{0}^{t-x_{1}} \cdots \int_{0}^{t-x_{1}-\cdots-x_{n}} \mathrm{~d} F(u) \mathrm{d} F\left(x_{n}\right) \cdots \mathrm{d} F\left(x_{1}\right)=B F_{n+1}(t),
$$

where $F_{n}$ is the ordinary convolution of $F$ with itself $n$ times. Letting $n \rightarrow \infty$ we see that $R(F, t)=0$ for every $t$, and this completes the proof. 


\subsection{Asymptotic behavior of solutions}

In ordinary renewal theory, the known asymptotic behavior of solutions of renewal-type integral equations (see, for instance, Section 5.6 of [7]) provides a powerful tool for understanding the long-term behavior of operating characteristics of systems that such equations model. Similar asymptotic results are obtainable for semi-renewal processes as well, and we explore this in this section. We first prove a technical result.

Lemma 1. Suppose that $m$ is a continuous function of bounded variation satisfying

$$
\lim _{t \rightarrow \infty}[m(t)-m(t-a)]=\omega(a) \text { for all } a>0,
$$

and suppose that $\lim _{t \rightarrow \infty} a(t)=$ A. Then

$$
\lim _{t \rightarrow \infty}[m(t)-m(t-a(t))]=\omega(A) .
$$

Proof. It suffices to show this for $m$ nondecreasing. We have $\lim _{t \rightarrow \infty} a(t)=A$ if and only if, for every $\varepsilon>0$, there is a $B=B(\varepsilon)$ such that $t \geq B$ entails $A-\varepsilon<a(t)<A+\varepsilon$. Set $j(t, a)=m(t)-m(t-a)$. Then $\lim _{t \rightarrow \infty} j(t, a)=L$ if and only if, for every $\varepsilon>0$, there is a $K=K(\varepsilon, a)$ such that $t>K$ entails $|j(t, a)-L|<\varepsilon$. Now choose $\varepsilon>0$ and take $t>\max \{B(\varepsilon), K(\varepsilon, \omega(A)-\varepsilon), K(\varepsilon, \omega(A)+\varepsilon)\}$. Then, for such $t, j(t, \omega(A)-\varepsilon)<$ $j(t, a(t))<j(t, \omega(A)+\varepsilon)$. But then, as $t \rightarrow \infty$, the left-hand side converges to $\omega(A-\varepsilon)$ and the right-hand side converges to $\omega(A+\varepsilon)$. Since $\varepsilon$ is arbitrary, the result follows.

The asymptotic behavior of (8) for the semi-renewal process depends on the tail properties of $M(F, t)$ and is contained in the following result.

Theorem 5. Suppose that $T_{u}$ satisfies the conditions of Theorem 2 and that

1. $\lim _{t \rightarrow \infty}[M(F, t)-M(F, t-a)]=\omega(a)$ for all $a>0$;

2. for each $t>0, h\left(T_{u} F, t-u\right)$ is nonincreasing as a function of $u \in[0, t]$;

3. $h_{0}(t):=h\left(T_{t} F, 0\right)<\infty$ for all $t, \lim _{t \rightarrow \infty} h_{0}(t)=\eta_{0}>0$, and $\lim _{t \rightarrow \infty} h(F, t)=0$;

4. $\lim _{t \rightarrow \infty} \int_{0}^{t} h\left(T_{u} F, t-u\right) \mathrm{d} u$ exists.

Then, for the solution (8) $r(F, t)$ of (7), we have

$$
\lim _{t \rightarrow \infty} r(F, t)=\lim _{t \rightarrow \infty} \int_{0}^{t} \omega(t-u) \mathrm{d}_{u} h\left(T_{u} F, t-u\right) .
$$

Proof. Define $\{Y(t): t \geq 0\}$ by

$$
\mathrm{P}\{Y(t) \leq y\}=\left[1-\frac{h\left(T_{t-y} F, y\right)}{h_{0}(t)}\right] V(t-y) .
$$

Note that

$$
\mathrm{P}\{Y(t)>y\}=\frac{h\left(T_{t-y} F, y\right)}{h_{0}(t)} \quad \text { for } 0 \leq y \leq t .
$$


Choose $S>0$, and set $Y_{S}(t)=Y(t) V(S-t)+Y(S) V(t-S)$. Then

$$
\begin{aligned}
r(F, t) & =h(F, t)+\int_{0}^{t} h\left(T_{y} F, t-y\right) \mathrm{d} M(F, y) \\
& =h(F, t)+h_{0}(t) \int_{0}^{t} \mathrm{P}\left\{Y_{S}(t)>t-y\right\} \mathrm{d} M(F, y) \\
& =h(F, t)+h_{0}(t) \int_{0}^{t} \mathrm{E} V\left(Y_{S}(t)-t+y\right) \mathrm{d} M(F, y) \\
& =h(F, t)+h_{0}(t) \mathrm{E}\left[\int_{0}^{t} V\left(Y_{S}(t)-t+y\right) \mathrm{d} M(F, y)\right] \\
& =h(F, t)+h_{0}(t) \mathrm{E}\left[\int_{t-Y_{S}(t)}^{t} \mathrm{~d} M(F, y)\right] \\
& =h(F, t)+h_{0}(t) \mathrm{E}\left[M(F, t)-M\left(F, t-Y_{S}(t)\right)\right] .
\end{aligned}
$$

It follows that

$$
\lim _{t \rightarrow \infty} r(F, t)=\eta_{0} \lim _{t \rightarrow \infty} \mathrm{E}\left[M(F, t)-M\left(F, t-Y_{S}(t)\right)\right]
$$

Noting that $\lim _{t \rightarrow \infty} Y_{S}(t)=Y(S)$ almost surely and applying Lemma 1, we obtain

$$
\lim _{t \rightarrow \infty}\left[M(F, t)-M\left(F, t-Y_{S}(t)\right)\right]=\omega(Y(S))
$$

with probability 1 . Also, $0 \leq M(F, t)-M\left(F, t-Y_{S}(t)\right) \leq M(F, t)$ because $M$ is nonnegative and nondecreasing. As $M(F, t)$ is integrable $(\mathrm{P})$, we can interchange the limit and expectation to obtain

$$
\begin{aligned}
\lim _{t \rightarrow \infty} \mathrm{E}\left[M(F, t)-M\left(F, t-Y_{S}(t)\right)\right] & =\mathrm{E} \lim _{t \rightarrow \infty}\left[M(F, t)-M\left(F, t-Y_{S}(t)\right)\right] \\
& =\mathrm{E} \omega(Y(S)),
\end{aligned}
$$

so that

$$
\begin{aligned}
\lim _{t \rightarrow \infty} r(F, t) & =\eta_{0} \int_{0}^{\infty} \omega(u) \mathrm{dP}\{Y(S) \leq u\} \\
& =-\frac{\eta_{0}}{h_{0}(S)} \int_{0}^{S} \omega(u) \mathrm{d}_{u} h\left(T_{S-u} F, u\right) \\
& =\frac{\eta_{0}}{h_{0}(S)} \int_{0}^{S} \omega(S-u) \mathrm{d}_{u} h\left(T_{u} F, S-u\right) .
\end{aligned}
$$

But $S$ is arbitrary, so we finally obtain

$$
\lim _{t \rightarrow \infty} r(F, t)=\lim _{t \rightarrow \infty} \int_{0}^{t} \omega(t-u) \mathrm{d}_{u} h\left(T_{u} F, t-u\right) .
$$

This completes the proof. 
For example, if $T_{u}=I$ and the semi-renewal process is a renewal process, then $\omega(a)=a / \mu$, where $\mu$ is the mean of $F$. Then Theorem 5 is the well-known result [7, pp. 184-185]

$$
\begin{aligned}
\lim _{t \rightarrow \infty} r(F, t) & =\frac{1}{\mu} \lim _{t \rightarrow \infty} \int_{0}^{t}(t-u) \mathrm{d}_{u} h(F, t-u) \\
& =\frac{1}{\mu} \lim _{t \rightarrow \infty} \int_{0}^{t} t \mathrm{~d}_{u} h(F, t) \\
& =\frac{1}{\mu} \int_{0}^{\infty} h(F, x) \mathrm{d} x,
\end{aligned}
$$

upon integration by parts. That $\omega(a)=c a$, as in the renewal process, is not true in general; for an example, use the record value process with $F(t)=1-\exp (-\lambda t)$, for which $M(F, t)=$ $\exp (\lambda t)-1$ and $\omega(a)=[1-\exp (-\lambda a)] \exp (\lambda t)$.

A more useful form of the asymptotic result is given in the following corollary.

Corollary 1. Suppose that the conditions of Theorem 5 are satisfied, except that condition 2 is replaced by

- for $t>0, h\left(T_{u} F, t-u\right)$ is of bounded variation as a function of $u \in[0, t]$ and is locally semi-bounded.

Then (10) holds.

Proof. Since $h\left(T_{u} F, t-u\right)$ is of bounded variation as a function of $u \in[0, t]$ and is locally semi-bounded, we may write it as the difference of two nonincreasing functions (take the usual representation as the difference of two increasing functions $f-g$, then use the local semi-boundedness bound $B$ to write as $B-g-(B-f)$ ), say $h_{1}$ and $h_{2}$. Set

$$
r_{i}(F, t)=h_{i}(F, t)+\int_{0}^{t} h_{i}\left(T_{u} F, t-u\right) \mathrm{d} M(F, u) \quad \text { for } i=1,2,
$$

and note that

$$
\lim _{t \rightarrow \infty} r_{i}(F, t)=\lim _{t \rightarrow \infty} \int_{0}^{t} \omega(t-u) \mathrm{d}_{u} h_{i}\left(T_{u} F, t-u\right),
$$

by Theorem 5. Since $r=r_{1}-r_{2}$, the result follows.

\subsection{Example: forward recurrence times}

To illustrate the applicability of the results of the previous two sections, we will derive the distribution and asymptotic distribution of the forward recurrence times in a semi-renewal process, and, as a result, obtain these for the revival process and the record value process in a novel way.

Denote by $\gamma_{t}(F)$ the time from $t \geq 0$ to the next event in a semi-renewal process corresponding to the semigroup $T$ and having distribution $F$ of the time to its first event, and set $q_{t}(F, y)=\mathrm{P}\left\{\gamma_{t}(F)>y\right\}$. Then, using Theorem 1 , we obtain

$$
\mathrm{P}\left\{\gamma_{t}(F)>y \mid X_{1}=u\right\}= \begin{cases}1, & u-t>y, \\ 0, & u-t \leq y, \\ \mathrm{P}\left\{\gamma_{t-u}\left(T_{u} F\right)>y\right\}, & t \geq y,\end{cases}
$$


which leads directly to the semi-renewal integral equation

$$
q_{t}(F, y)=1-F(y+t)+\int_{0}^{t} q_{t-u}\left(T_{u} F, y\right) \mathrm{d} F(u) .
$$

In the notation of Section 4.2 we have $h(F, t)=1-F(y+t)$. From the general solution (8) we immediately obtain

$$
q_{t}(F, y)=1-F(y+t)+\int_{0}^{t}\left[1-T_{u} F(y+t-u)\right] \mathrm{d} M(F, u) .
$$

For the revival process where $T$ is given by (3), (11) reduces to

$$
q_{t}(F, y)=1-F(y+t)+\int_{0}^{t} \frac{1-F(y+t)}{[1-F(u)]^{2}} \mathrm{~d} F(u)=\frac{1-F(y+t)}{1-F(t)}=1-T_{t} F(y),
$$

so that, finally, $\mathrm{P}\left\{\gamma_{t}(F) \leq y\right\}=T_{t} F(y)$, which is a familiar result for the nonhomogeneous Poisson process [10] now shown to also hold for the revival process.

In the case of the record value process, whose semigroup is given by $T_{u} F(x)=F(x+u)$, (11) reduces to

$$
\begin{aligned}
q_{t}(F, y) & =1-F(y+t)+\int_{0}^{t}[1-F(y+t)] \mathrm{d}\left[\frac{F(u)}{1-F(u)}\right] \\
& =[1-F(y+t)]\left[1+\frac{F(t)}{1-F(t)}\right] \\
& =1-T_{t} F(y),
\end{aligned}
$$

which is again a familiar result for the record values process [11].

In these two examples, the forward recurrence time distributions are the same, and the asymptotic analysis easily follows from

$$
\lim _{t \rightarrow \infty} \mathrm{P}\left\{\gamma_{t}(F) \leq y\right\}=\lim _{t \rightarrow \infty} \frac{F(y+t)-F(t)}{1-F(t)},
$$

to which we can apply, e.g. the theory of regularly varying functions [6, pp. 275ff.] or other asymptotic properties of $F$ that may apply.

\section{Summary and conclusions}

We have defined the framed point process as a model of 'reliability prediction' that explicitly captures assumptions about how the future is connected to the past. For the semi-renewal special case of framed point processes, we have shown that familiar results about renewal processes extend and generalize, showing along the way a unification of the renewal and revival processes via the property that all the one-dimensional distributions of these processes are fully determined by the distribution of the time to the initial event.

\section{References}

[1] Ascher, H. E. And Feingold, H. (1984). Repairable Systems Reliability. Marcel Dekker, New York.

[2] BAXter, L. A. AND Chlouverakis, G. (1990). A model for a repairman who introduces faults. Working paper, Stern School of Business, New York University. 
[3] Baxter, L. A. And Chlouverakis, G. (1990). Optimal maintenance with an imperfect repairman. Working paper, Stern School of Business, New York University.

[4] Baxter, L. A., Kijima, M. And Tortorella, M. (1996). A point process model for the reliability of a maintained system subject to general repair. Commun. Statist. Stoch. Models 12, 37-65.

[5] Brown, M. and Proschan, F. (1983). Imperfect repair. J. Appl. Prob. 20, 851-859.

[6] Feller, W. (1971). An Introduction to Probability Theory and Its Applications, Vol. II, 2nd edn. John Wiley, New York.

[7] Karlin, S. and Taylor, H. M. (1975). A First Course in Stochastic Processes, 2nd edn. Academic Press, New York.

[8] Kisima, M. (1989). Some results for repairable systems with general repair. J. Appl. Prob. 26, 89-102.

[9] KiJima, M. AND Sumita, U. (1986). A useful generalization of renewal theory: counting processes governed by nonnegative Markovian increments. J. Appl. Prob. 23, 71-88.

[10] Shanthikumar, J. G. and BaXter, L. A. (1985). Closure properties of the relevation transform. Naval Res. Logistics 32, 185-189.

[11] Shorrock, R. W. (1972). On record values and record times. J. Appl. Prob. 9, 316-326.

[12] Snyder, D. L. (1975). Random Point Processes. John Wiley, New York.

[13] Tortorella, M. (2010). Strong and weak minimal repair and the revival process in reliability theory. Submitted.

[14] Yosida, K. (1971). Functional Analysis, 3rd edn. Springer, New York. 